\title{
Impacts of gradual automated vehicle penetration on motorway operation: a comprehensive evaluation
}

\author{
Duo $\mathrm{Li}^{1,2^{*}}$ (D) and Peter Wagner ${ }^{2}$
}

\begin{abstract}
Gradual penetration of automated vehicles (AVs) into current motorway systems will usher the stage of mixed traffic in which AVs will coexist with human driven vehicles. Thus, there is an urgent need to identify the possible impacts of this mixed traffic on motorway operation. To investigate the potential benefits or losses due to introducing AVs into existing motorway systems, this study conducts a comprehensive evaluation based on simulation using a $5.3 \mathrm{~km}$ stretch on Auckland Motorway and traffic data provided by New Zealand Traffic Agent (NZTA). We analyze the impacts of different AV shares on mobility, safety, emissions and fuel consumption. The motorway with and without traffic control are tested under four scenarios of traffic conditions, namely, heavily congested traffic $\left(>0.95^{*}\right.$ capacity), lightly congested traffic $\left(\approx 0.7^{*}\right.$ capacity), free-flow traffic $\left(\approx 0.5^{*}\right.$ capacity), and future traffic ( $3^{*}$ heavily congested traffic volume). The outcomes of the research can provide motorway designers and operators a reasonable range of influences contributed by AV penetration so as to better prepare for AVs' arrival.
\end{abstract}

Keywords: Automated vehicle, Motorway operation, Micro-simulation

\section{Introduction}

Recent advances in vehicle automation have opened up new perspectives for the design and operation of motorway systems. The National Highway Transportation Safety Administration [32] has classified five levels of automated vehicle (AV) functionality, ranging from no AV features (level 0) to full automation without human controls (level 4). Semi-automation (levels 1 to 3 ), i.e., parking assist systems, adaptive cruise control (ACC), collision avoidance, lane departure warnings, are now commercially available in products of major auto manufacturers [12]. Level 4 AVs are already driving on the roads, but are not yet available for the normal customer. With such technology becoming successful and available to the mass market, it is likely to have direct impacts on motorway operation.

Existing studies have identified positive achievements of AVs in mobility, safety, emissions and travel behavior. For example, Tientrakool et al. [37] observed 43\% increase in motorway capacity because of AVs. Hayes [14]

\footnotetext{
* Correspondence: duoli0725@gmail.com

${ }^{1}$ School of Highway, Chang'an University, Xi'an 710064, Shanxi, China ${ }^{2}$ Institute of Transport Systems, German Aerospace Center (DLR), 12489 Berlin, Germany
}

suggested that fatality rates could eventually approach $1 \%$ of current rates when $100 \%$ AVs is realized. Brown et al. [4] and Morrow et al. [30] estimated that AVs could reduce fuel consumption up to $80 \%$. Obviously, such significant improvements were estimated based on the optimistic assumption that high AV shares are present. However, realizing a $95 \%$ or more AV shares will be a long-term goal. At the initial stage of deployment, AVs will coexist with human driven vehicles (HDVs) on motorways. AVs' gradual penetration into the current motorway systems will usher the stage of mixed traffic before they become universal.

A number of studies [3, 24, 36] have been performed to investigate the impact of changes in AV penetration on traffic flow. Most evaluation studies focused on single lane traffic taking little consideration of motorway layout features (e.g., merging areas, lane reduction) and lane changing behaviors into account. Furthermore, existing studies focused on the issues of stability and congestion, whereas the safety and environmental impacts of AVs have been rarely touched. Meanwhile, there exists a considerable discrepancy in the body of literature on mobility gains or losses due to the introduction of AVs. Some 
researchers concluded that introducing AVs could relieve or even eliminate congestion $[24,36]$. In contrast, Davis [8] observed that the formation of congestion was sensitive to the sequence of vehicles and AVs with higher speeds were more likely to result in jams. Jerath and Brennan [20] stated that though more AVs could provide higher flows, they also cause disproportionately higher susceptibility to congestion.

The main goal of motorway traffic management is to improve efficiency of motorways under acceptable safety standards. Variable Speed Limit (VSL) is most commonly used to achieve such a goal. Through better harmonization of traffic flows, the VSL is effective to improve traffic safety and reduce traffic congestion and emissions [10, 19, 27]. The mixed traffic flow where AVs and HDVs coexist may dramatically change the performance of existing VSL systems because of the following reasons. Firstly, the success of VSL is highly dependent on the level of drivers' compliance [27]. AVs programmed to not break speed limits have the potential to enhance the effectiveness of existing VSL systems. Secondly, the underlying concepts of VSL and AV for improving mobility are different. The former relieves congestion via reducing and harmonizing traffic flow, whereas the latter encourages smaller time gaps between vehicles so as to increase motorway capacity and prevent jams.

A number of studies have been conducted to investigate the performance of traffic control measures (e.g., VSL) at various AV market shares; most of them focused on mobility changes caused by AV penetration. For example, Kesting et al. [23] assessed the impact of various ACC strategies on the bottleneck capacity at AV penetration rates ranging between $0 \%$ and $50 \%$. Müller et al. [31] studied the influence of VSL on vehicle delay when $0 \%-100 \%$ AVs were deployed. Using total time spend (TTS) as the performance measure, Perraki et al. [35] tested their proposed VSL and ramp metering strategies in $20 \%, 50 \%$ and $100 \%$ AV cases. Nevertheless, there are limited works that comprehensively assess the AV impact on VSL in terms of mobility, safety, emissions and fuel consumption at various penetration rates. For example, Khondaker and Kattan [22] evaluated mobility, safety and environmental performance of their proposed VSL algorithm. The authors only considered two cases, namely, $50 \%$ and $100 \%$ AVs cases, whereas it might be more interesting to identify the possible impacts of AVs in the near futures (e.g., 10\% AVs are introduced).

In this research, a comprehensive evaluation of AVs' potential impacts on an existing motorway system is presented. A $5.3 \mathrm{~km}$ stretch on Auckland Motorway State Highway 16 (SH 16) that connects Central Auckland with Northwestern Auckland is simulated based on traffic data provided by New Zealand Transport Agency (NZTA). We investigate the AVs' influence on various motorway operational scenarios including heavily congested traffic ( $>0.95^{*}$ capacity), lightly congested traffic $\left(\approx 0.7^{*}\right.$ capacity), free-flow traffic $\left(\approx 0.5^{*}\right.$ capacity), and future traffic (3*heavily congested traffic volume) conditions. The motorway with and without traffic control are tested. A number of measures of effectiveness (MoEs) are computed to reflect the changes in mobility, safety, fuel consumption and emissions caused by the deployment of AVs. The outcomes of the research can provide motorway designers and operators a reasonable range of influences contributed by AV penetration so as to better prepare for AVs' arrival.

\section{Methods}

In this research, we used a microscopic traffic simulation approach. Since AVs are not available to the mass market yet, assessing the impacts of AV penetration on motorway systems in the field is not an option.

\subsection{Description of the test bed}

As a test bed for this study, a critical bottleneck section on Auckland Motorway SH16 connecting northwestern Auckland with central Auckland (see Fig. 1) was selected and simulated using SUMO micro-simulator [2, 25]. The $5.3 \mathrm{~km}$ motorway stretch consists of multiple on-off ramps, with speed limit of $100 \mathrm{~km} / \mathrm{h}$. The default maximum speed limit on expressways and motorways in New Zealand is

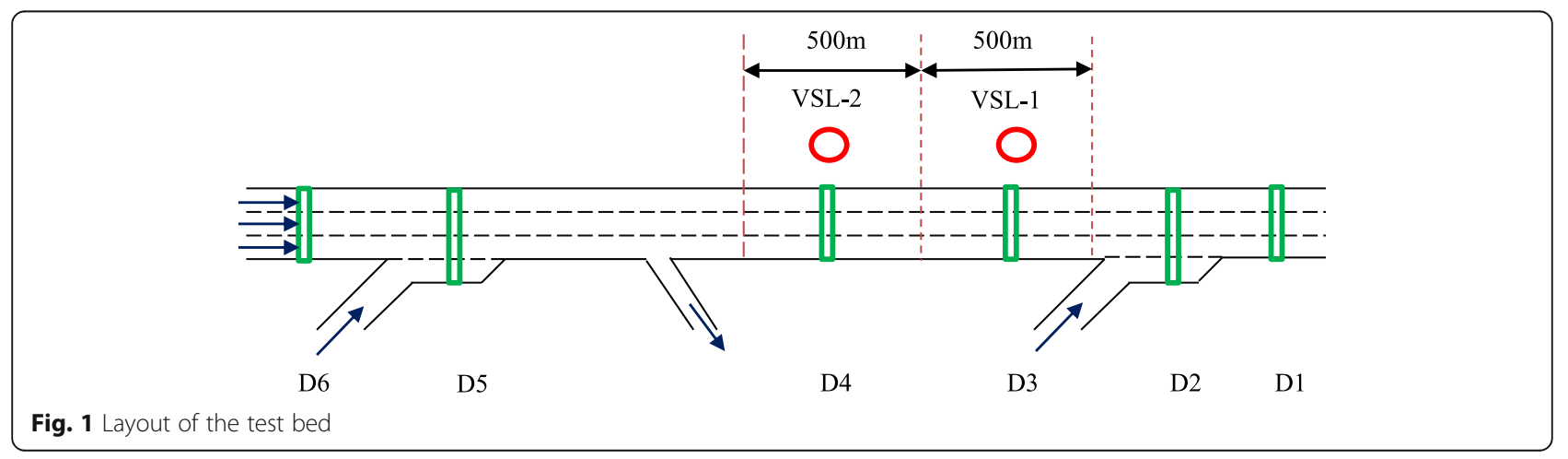


$100 \mathrm{~km} / \mathrm{h}$. In New Zealand, speed limit signs follow the European model of a number inside a red circle.

NZTA provided the motorway traffic data including vehicle count and occupancy data accumulated over a 30-s period. Data for the selected motorway were obtained for a period of 3 months, from 5 March 2012 to 27 May 2012. Two typical weekdays, March 12 (Monday) and 9 (Friday) were chosen to calibrate and validate the model. The GEH value [9] was used to measure the goodness of the calibration and validation processes:

$$
\mathrm{GEH}=\sqrt{\frac{(E-F)^{2}}{(E+F) / 2}}
$$

where, E is estimated count using SUMO model, and F is field count. Note that 5-min vehicle counts from the simulation outputs and real world measurements were used to compute GEH values of 9 different locations. For calibration, all the locations obtained GEH $<5$ for more than $85 \%$ cases and $\mathrm{GEH}<4$ was achieved for the sum of all traffic volumes of all segments. For validation, 8 locations and 1 location produced $\mathrm{GEH}<5$ and $\mathrm{GEH}<8$ for more than $85 \%$ cases respectively; $\mathrm{GEH}<4$ was recorded for the sum of all traffic volumes. The calibration and validation results indicate that the developed model is acceptable.

\subsection{Simulating AV and HDV}

We used the default car following model [26] of SUMO. The concept of the Krauß model is that the desired vehicle speed is the minimum of 1) the maximum speed of the vehicle, 2) the displayed speed limit, 3) the speed using the vehicle's maximum acceleration ability and 4) a safe speed resulting in no collision. The Krauß model assumes that the driver is not perfect in holding its desired speed. The imperfection of the driver is modelled as a stochastic deceleration. An AV is assumed to have a smaller time-gap, a lower driver imperfection and a higher compliance rate to speed limits when compared against a HDV. Thus, we set the minimum time-gap $T=0.5 \mathrm{~s}(\mathrm{AV}) / 1.1 \mathrm{~s}(\mathrm{HDV})$, the driver imperfection $d_{a}=$ $0(\mathrm{AV}) / 0.5(\mathrm{HDV})$ and the speed factor $d_{v}=0.01(\mathrm{AV})$ / 0.15 (HDV). Note that $T$ is the minimum possible time-gap that drivers attempt to maintain in order to assure the possibility to brake in time. The lower driver imperfection value (between 0 and 1 ) leads to more accurate acceleration actions. The speed factor $d_{v}$ is the coefficient of variance of the desired speed. For example, $d_{v}=0.15$ will result in a speed distribution where $95 \%$ of the vehicles drive between $70 \%$ and $130 \%$ of the legal speed limit.

We selected the lane-changing model developed by Erdmann [11]. Compared to other lane-changing models, the model by Erdmann [11] explicitly discriminates between four different motivations for lane- changing: 1) strategic change, 2) cooperative change, 3) tactical change and 4) regulatory change. A vehicle performs strategic changes in order to avoid reaching a "dead" lane on which the vehicle is not able to continue its pre-defined route. A cooperative change is performed by the vehicle with the purpose of helping another vehicle with lane-changing towards its lane. Tactical change occurs when a vehicle attempts to avoid following a slow leader. A vehicle performs a regulatory change in order to clear the overtaking lane. With the advanced vehicle communication technologies, AVs are assumed to have higher willingness for performing cooperative lane changing than HDVs. Thus, we set the willingness for cooperative changing (between 0 and 1) as 0.99 and 0.7 for AVs and HDVs respectively.

\subsection{VSL control algorithm}

Existing VSL control algorithms can be broadly classified into four categories, namely, rule-based $[1,10,15,33$, $38]$, fuzzy-logic based [6, 28], analytical [16, 18] and control-theory based $[7,19,21]$ algorithms. Most of the VSL systems that are in operation employ rule based algorithms; they appear in many European countries, North America and elsewhere. In this study, we modified a rule based VSL algorithm which is in operation on I-4 in Orlando, United States [10]. Compared to other measurements used for rule-based algorithms, the occupancy provides a more robust and efficient target for VSL operation. This is because traffic flow capacity values may vary on different days, even under similar environmental conditions; while the critical occupancy values are more stable from day to day [5, 34].

In the existing I-4 algorithm, traffic conditions are classified as either free-flow, light congestion, or heavy congestion based on pre-defined thresholds to determine an appropriate speed limit for the respective traffic condition. We optimized the thresholds and corresponding speed limits (see Fig. 2) based on simulation outputs. Different AVs market shares may lead to a different critical occupancy. Therefore, we fine-tuned the thresholds (p1-p5) for various AVs shares and the combination that yielded the shortest mean travel time was chosen. The modified I-4 algorithm is illustrated in Fig. 2. Note, that the VSL control interval is $5 \mathrm{~min}$. We have tested other control intervals and found 5 mins a good compromise. Shorter intervals lead to a slightly better performance, however the difference between 5 mins and 1 mins is less than $1 \%$ in the travel times. VSLs should not change to quickly in order to not worry the drivers too much.

\section{Results and discussion}

In SUMO, we systematically tested four different traffic scenarios for the study section, including heavily congested, lightly congested scenario, non-congested and 




Fig. 2 VSL control logic

future scenarios. The simulation period and warm-up period of each scenario are $60 \mathrm{~min}$ and $15 \mathrm{~min}$ respectively. For the first three scenarios, mainline and onramp inflows are assigned based on the real traffic data collected from the detector D6 and the on-ramp detectors respectively. Two emission models are available in SUMO, one that is based on the German HBEFA (the older one), and one that is based on PHEM from the TU
Graz and is named PHEMlight in SUMO. The idea is the same, based on interpolation they assign to the acceleration and speed in each time-step the emission values. The difference is in the tables behind these two: the HBEFA tables are a kind of average over many different vehicles and different traffic scenarios, while the PHEM data-base is much cleaner. A good description can be found in [13]. The simulation results shown here

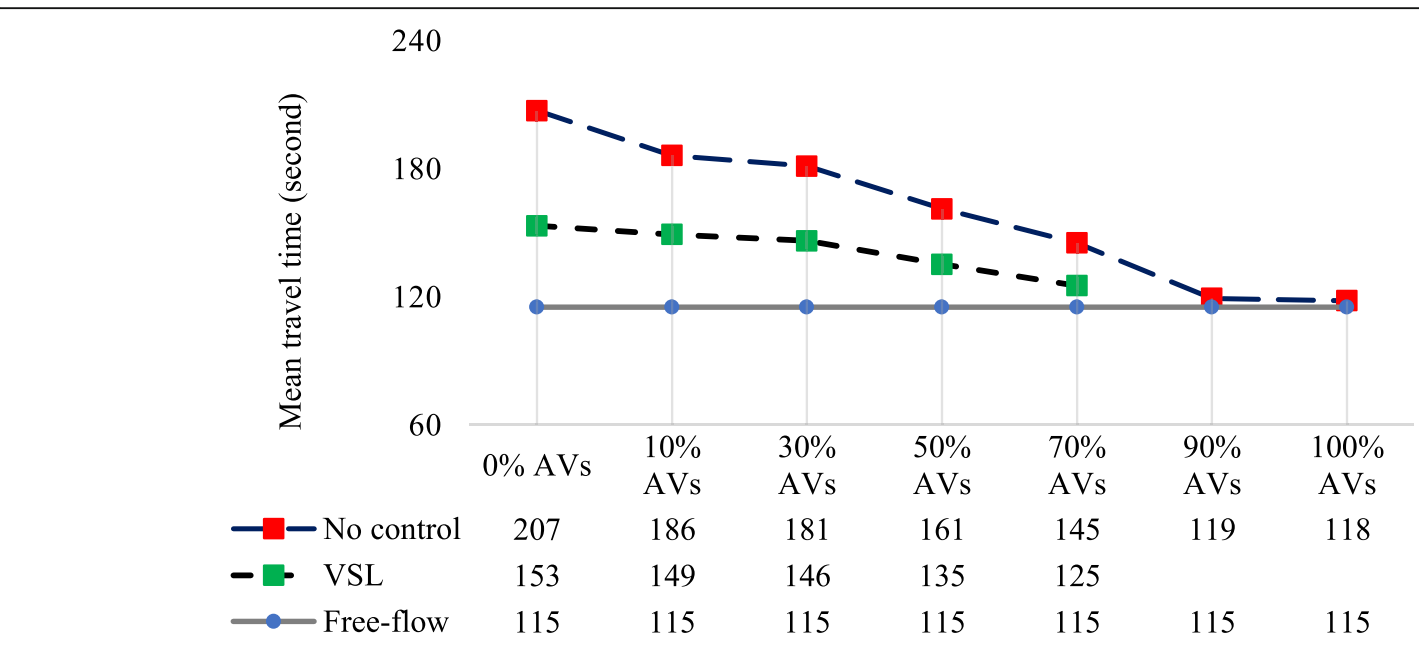

Fig. 3 Mean travel time for different AV penetration rates under heavily congested traffic 
60



Fig. 4 The number of $T T C<5 s$ under heavily congested Traffic

are based on the HBEFA approach. It should be noted that we have tested both models in a few examples, the PHEMlight produced slightly higher, but not totally different results. Note also, that the simulation results depend on the interplay between the microscopic model and the emission model. Since autonomous driving smooth traffic flow, and thereby reduce accelerations, it is understandable that emissions go down with AV's even in free traffic. The simulation results also showed that speed variations can be reduced by increasing AVs. The fact that the human driving model is more stochastic than the AV model may also play a role here.

\subsection{Heavily congested traffic (mean volume > $0.95 *$ capacity, 5-6 pm)}

In this study, mean travel time (TT) is used to measure the mobility gains of AVs. The simulation results show that the free-flow TT is about $115 \mathrm{~s}$. Note that the average travelled distance of each vehicle was about $3.2 \mathrm{~km}$. Figure 3 presents the TT values for different AV penetration rates under heavily congested traffic conditions. For no control cases, it is observed that higher AV penetration rates result in lower TT values. About 10\% and 12\% improvements in TT values are witnessed at $10 \%$ and $30 \%$ AV penetration rates. When $50 \%$ and $70 \%$ AVs are present, $18 \%$ and $30 \%$ improvements due to AV penetration are recorded compared to the $0 \% \mathrm{AVs}$ case.
However, the reduction in TT is found to be negligible in magnitude when more than $90 \%$ AVs appear on motorways. The implementation of VSL exerts positive impact on the mixed traffic flow when inserting 0-70\% AVs. The VSL is not triggered at all at $90 \%$ and $100 \%$ AVs market shares.

Time to collision (TTC) is used to measure the safety benefits attributed by AVs. Hayward [17] defined TTC as: "The time required for two vehicles to collide if they continue at their present speed and on the same path". Though no well-established consensus can be reached about critical value of TTC, it is proposed for safety studies that this value should be between 1.5 and $5 \mathrm{~s}$ [29]. Thus the number of TTC $<5 \mathrm{~s}$ is calculated and presented in Fig. 4. At the initial stage of deployment (10\% and $30 \% \mathrm{AVs})$, there is negative impact on motorway safety because of the introduction of AVs. The least safe situation is observed at $30 \% \mathrm{AV}$ penetration rate. The number of TTC $<5 \mathrm{~s}$ can be reduced to 2 and 1 at $90 \%$ and $100 \%$ AV shares respectively. VSL shows the ability to improve the safety of the mixed traffic flow. The negative impact of low AV shares on safety is partially relieved by applying VSL.

Emissions and fuel consumption computed using heavily congested traffic conditions are shown in Table 1. It can be clearly observed that emissions and fuel consumption are reduced by introducing AVs. The

Table 1 Emissions and fuel consumption under heavily congested traffic

\begin{tabular}{|c|c|c|c|c|c|c|c|c|c|c|c|c|}
\hline \multirow[t]{3}{*}{ AVs share } & \multirow{3}{*}{$\begin{array}{l}\text { Baseline } \\
0 \%\end{array}$} & \multicolumn{11}{|c|}{ Improvement } \\
\hline & & \multicolumn{6}{|c|}{ No control } & \multicolumn{5}{|c|}{ VSL control } \\
\hline & & $10 \%$ & $30 \%$ & $50 \%$ & $70 \%$ & $90 \%$ & $100 \%$ & $0 \%$ & $10 \%$ & $30 \%$ & $50 \%$ & $70 \%$ \\
\hline $\mathrm{CO}(\mathrm{mg})$ & $6.2 \times 10^{7}$ & $12 \%$ & $16 \%$ & $31 \%$ & $43 \%$ & $64 \%$ & $69 \%$ & $35 \%$ & $36 \%$ & $41 \%$ & $53 \%$ & $67 \%$ \\
\hline $\mathrm{CO} 2$ (mg) & $3.2 \times 10^{9}$ & $5 \%$ & $9 \%$ & $17 \%$ & $25 \%$ & $39 \%$ & $44 \%$ & $10 \%$ & $14 \%$ & $17 \%$ & $25 \%$ & $33 \%$ \\
\hline $\mathrm{HC}(\mathrm{mg})$ & $3.6 \times 10^{5}$ & $11 \%$ & $15 \%$ & $27 \%$ & $39 \%$ & $58 \%$ & $64 \%$ & $28 \%$ & $31 \%$ & $36 \%$ & $45 \%$ & $58 \%$ \\
\hline PMx (mg) & $6.8 \times 10^{4}$ & $5 \%$ & $10 \%$ & $17 \%$ & $26 \%$ & $41 \%$ & $47 \%$ & $10 \%$ & $12 \%$ & $18 \%$ & $25 \%$ & $33 \%$ \\
\hline NOx (mg) & $1.3 \times 10^{6}$ & $6 \%$ & $11 \%$ & $19 \%$ & $29 \%$ & $45 \%$ & $51 \%$ & $11 \%$ & $15 \%$ & $20 \%$ & $27 \%$ & $38 \%$ \\
\hline Fuel (ml) & $1.4 \times 10^{6}$ & $5 \%$ & $9 \%$ & $17 \%$ & $25 \%$ & $39 \%$ & $44 \%$ & $10 \%$ & $14 \%$ & $18 \%$ & $24 \%$ & $31 \%$ \\
\hline
\end{tabular}






Fig. 5 Mean travel time for different AV penetration rates under lightly congested traffic

environmental performance is further improved by applying VSL. This amelioration is mainly contributed by improved traffic conditions.

\subsection{Lightly congested traffic (mean volume $\approx 0.7^{*}$ capacity, 14:30-15:30 pm)}

Figure 5 presents the TT values under lightly congested traffic conditions. Lower TT values are achieved via introducing more AVs into the network. Either inserting AVs or applying VSL can lead to reduction in TT values when less than $30 \%$ AVs are present. Although at relatively high AV shares ( $\geq 50 \%)$ VSL is not activated at all, solely implementing AVs can yield low TT values which are close to free-flow TT.

Figure 6 presents the number of TTC $<5 \mathrm{~s}$ under lightly congested traffic conditions. Reduction in TTC is found to be not remarkable in magnitude at low AV shares (less than $30 \% \mathrm{AVs})$. However, this measure decreases dramatically by introducing $50 \%$ or more AVs. VSL also shows the ability to improve the safety of the mixed traffic flow. Meanwhile, Table 2 indicates that environmental gains of the motorway rise with the increase of AVs; Applying VSL control can further enhance this amelioration.

\subsection{Non congested traffic (mean volume $\approx 0.5^{*}$ capacity, 12:30-13:30 pm)}

Table 3 presents MoEs computed under non congested traffic conditions. No VSL control action is triggered in this scenario. The number of TTC $<5 \mathrm{~s}$ was 3 in $0 \%$ and $10 \%$ AVs cases, then reduced to 1 when more than $30 \% \mathrm{AVs}$ were deployed. Although reductions in TT, TTC, emissions and fuel consumption can be still observed, these improvements are relatively of smaller magnitude compared to heavily and lightly congested traffic scenarios.

\subsection{Future traffic (mean volume $\approx 3^{*}$ heavily congested volume)}

The results presented in Section 3.1 show that at relatively high AV shares, congestion is relieved or even prevented; this might be attributed to decreased time gaps between vehicles and a consequent increase in motorway

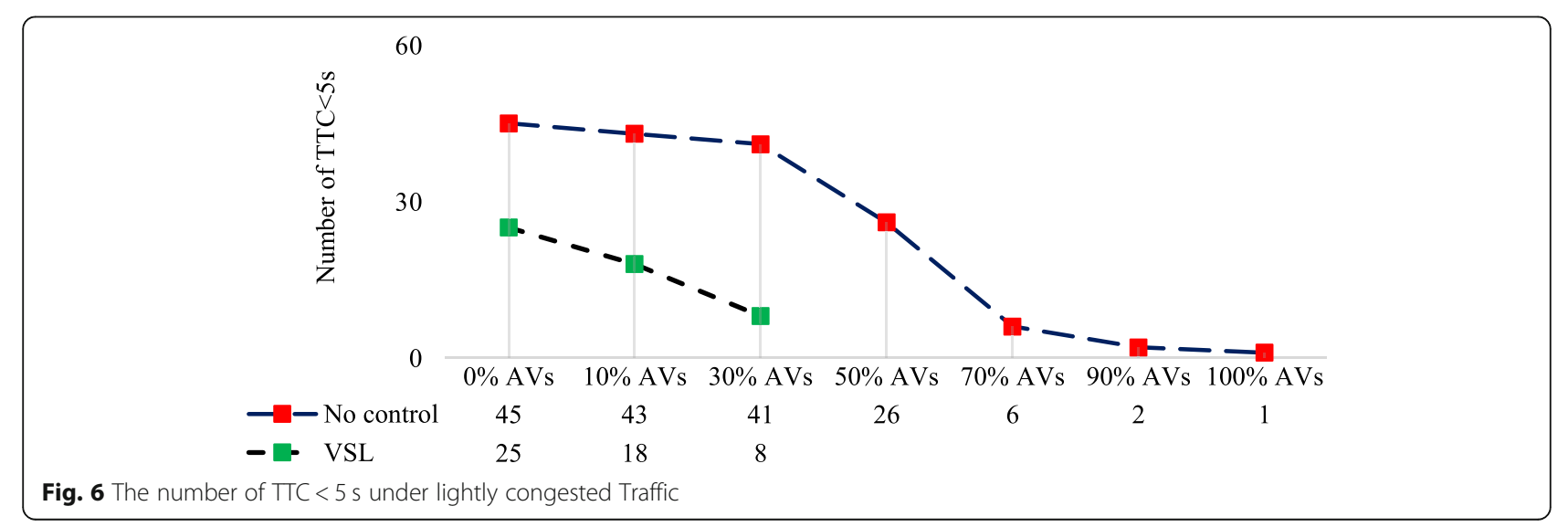


Table 2 Emissions and fuel consumption under lightly congested traffic

\begin{tabular}{|c|c|c|c|c|c|c|c|c|c|c|}
\hline \multirow[t]{3}{*}{ AVs share } & \multirow{3}{*}{$\begin{array}{l}\text { Baseline } \\
0 \%\end{array}$} & \multicolumn{9}{|c|}{ Improvement } \\
\hline & & \multicolumn{6}{|c|}{ No control } & \multicolumn{3}{|c|}{ VSL control } \\
\hline & & $10 \%$ & $30 \%$ & $50 \%$ & $70 \%$ & $90 \%$ & $100 \%$ & $0 \%$ & $10 \%$ & $30 \%$ \\
\hline $\mathrm{CO}(\mathrm{mg})$ & $4.2 \times 10^{7}$ & $2 \%$ & $7 \%$ & $16 \%$ & $27 \%$ & $36 \%$ & $41 \%$ & $23 \%$ & $38 \%$ & $48 \%$ \\
\hline $\mathrm{CO} 2$ (mg) & $2.5 \times 10^{9}$ & $2 \%$ & $10 \%$ & $26 \%$ & $43 \%$ & $53 \%$ & $58 \%$ & $6 \%$ & $14 \%$ & $20 \%$ \\
\hline $\mathrm{HC}(\mathrm{mg})$ & $2.5 \times 10^{5}$ & $1 \%$ & $7 \%$ & $17 \%$ & $29 \%$ & $40 \%$ & $45 \%$ & $18 \%$ & $31 \%$ & $41 \%$ \\
\hline PMx (mg) & $5.3 \times 10^{4}$ & $1 \%$ & $8 \%$ & $18 \%$ & $32 \%$ & $43 \%$ & $48 \%$ & $6 \%$ & $15 \%$ & $21 \%$ \\
\hline $\mathrm{NOx}(\mathrm{mg})$ & $9.7 \times 10^{5}$ & $1 \%$ & $7 \%$ & $16 \%$ & $27 \%$ & $36 \%$ & $41 \%$ & $7 \%$ & $15 \%$ & $23 \%$ \\
\hline Fuel (ml) & $1.1 \times 10^{6}$ & $5 \%$ & $9 \%$ & $17 \%$ & $25 \%$ & $39 \%$ & $44 \%$ & $6 \%$ & $14 \%$ & $21 \%$ \\
\hline
\end{tabular}

capacity. However, existing traffic demand cannot guarantee a fully utilization of this improved capacity. In order to explore the maximum possible ability of AVs to improve the existing motorway infrastructure, we increased the volume used in Section 3.1 by 3 times. Figure 7 demonstrates the maximum volumes and total throughputs under future traffic conditions. The motorway equipped with $100 \%$ AVs witnesses a significant increase in maximum volume (88\% improvement) and total throughputs (83\% improvement) compared against that with $0 \%$ AVs. Nevertheless, in this future scenario, the application of VSL slightly degrades the capacity of the selected motorway. At first, this is a bit surprising, but it is due to AV's driving already optimal. A VSL decreases their speed a bit, and with it, the throughput.

Table 4 presents MoEs computed using future traffic demand. Though increase in AV penetration still results in reduction in TT values, the motorway with VSL control produces a slightly higher TT than that without any control. With the increase of AVs, motorway safety drops. This might be resulted from extremely high traffic density and short time gaps between vehicles. Applying VSL aggravates this degradation. Introducing AVs also worsens the environmental performance; this is due an increase of the throughput. More specifically, the total number of vehicles loaded in this scenario was 15750 ; while the number of vehicles that entered the motorway was restricted by the throughput of the motorway (see
Fig. 7). The increase in emissions and fuel consumption due to additional vehicles is partially relieved using VSL.

\section{Conclusions}

In order to investigate the potential benefits or losses due to introducing AVs into the existing motorway systems, this study conducts a comprehensive evaluation based on simulation using a $5.3 \mathrm{~km}$ stretch on Auckland Motorway and traffic data provided by NZTA. We analyze the impacts of different AV shares on mobility, safety, driver behavior, emissions and fuel consumption of the selected motorway. The motorway with and without traffic control are tested under four scenarios of traffic conditions, namely, heavily congested traffic $\left(>0.95^{*}\right.$ capacity), lightly congested traffic $\left(\approx 0.7^{*}\right.$ capacity), free-flow traffic ( $\approx 0.5^{*}$ capacity), and future traffic (3*heavily congested traffic volume).

Under existing traffic conditions (first three scenarios), introducing AVs can improve the overall performance of the motorway infrastructure. More specifically, under lightly and heavily traffic conditions deploying more than $70 \%$ AVs can yield a mean travel time approaching that of free-flow. This is mainly attributed by the reduced congestion. In these two scenarios, congestion is prevented at relatively high AV penetration rates. This improved traffic conditions also contribute to the significant reduction in TTC, emissions and fuel consumption. Moreover, with the increase of AVs, drivers are more

Table 3 MoEs computed using non congested traffic

\begin{tabular}{|c|c|c|c|c|c|c|c|}
\hline \multirow[t]{2}{*}{ AV shares } & \multirow{2}{*}{$\begin{array}{l}\text { Baseline } \\
\text { 0\%AVs }\end{array}$} & \multicolumn{6}{|c|}{ Improvement } \\
\hline & & $10 \%$ AVs & $30 \%$ Avs & $50 \%$ Avs & 70\%Avs & $90 \%$ Avs & $100 \%$ Avs \\
\hline Mean TT & 122 & $0 \%$ & $3 \%$ & $4 \%$ & $5 \%$ & $6 \%$ & $6 \%$ \\
\hline $\mathrm{CO}(\mathrm{mg})$ & $2.1 \times 10^{7}$ & $3 \%$ & $15 \%$ & $23 \%$ & $34 \%$ & $45 \%$ & $50 \%$ \\
\hline $\mathrm{CO}_{2}(\mathrm{mg})$ & $1.6 \times 10^{9}$ & $2 \%$ & $10 \%$ & $16 \%$ & $24 \%$ & $31 \%$ & $35 \%$ \\
\hline $\mathrm{HC}(\mathrm{mg})$ & $1.4 \times 10^{5}$ & $2 \%$ & $14 \%$ & $21 \%$ & $31 \%$ & $41 \%$ & $46 \%$ \\
\hline PMx (mg) & $3.4 \times 10^{4}$ & $2 \%$ & $12 \%$ & $18 \%$ & $27 \%$ & $35 \%$ & $40 \%$ \\
\hline Nox (mg) & $6.0 \times 10^{5}$ & $2 \%$ & $12 \%$ & $19 \%$ & $29 \%$ & $37 \%$ & $42 \%$ \\
\hline Fuel (ml) & $6.7 \times 10^{5}$ & $2 \%$ & $10 \%$ & $16 \%$ & $24 \%$ & $31 \%$ & $35 \%$ \\
\hline
\end{tabular}



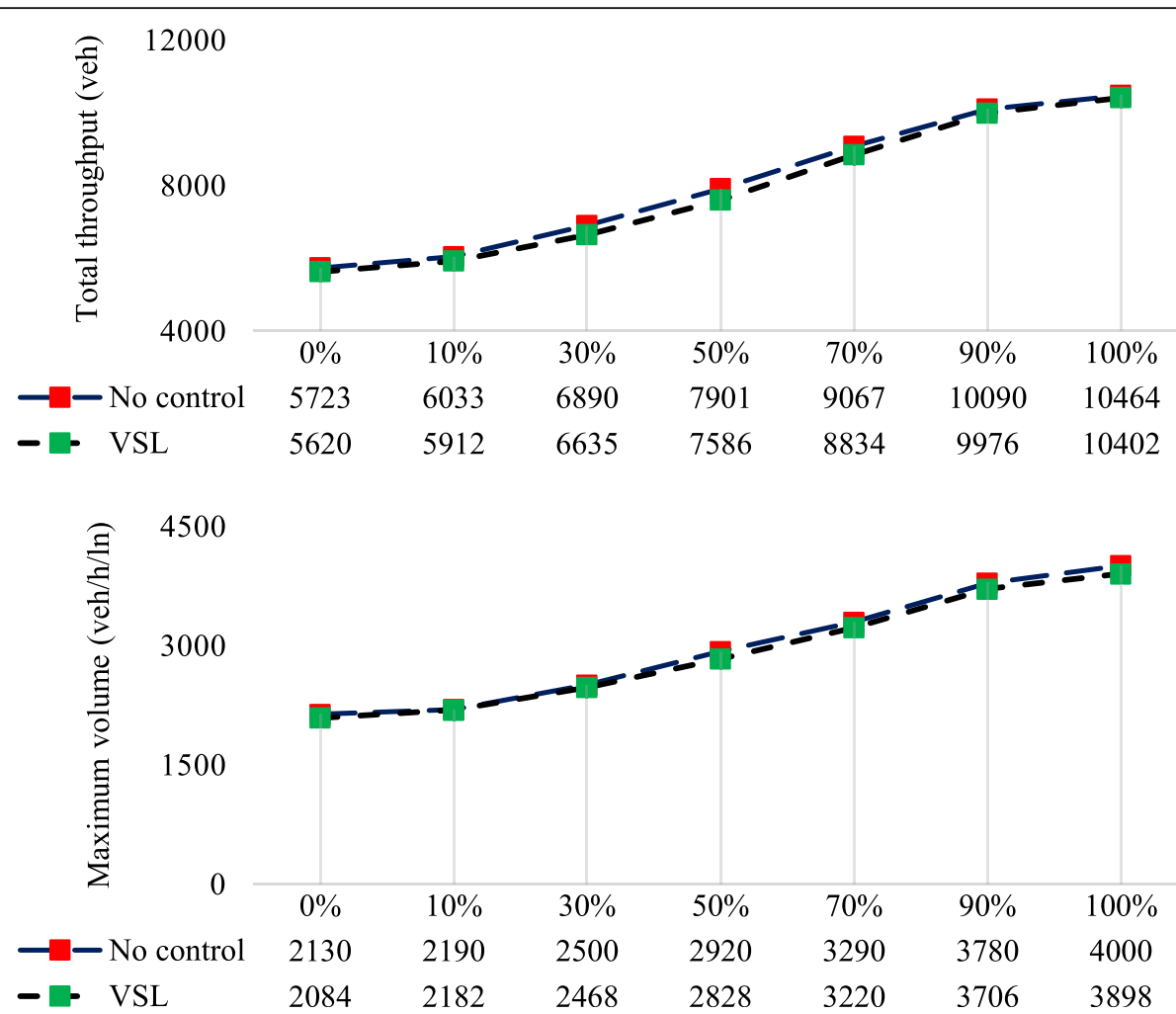

Fig. 7 Maximum volume and total throughput under future traffic

Table 4 MoEs computed using future traffic

\begin{tabular}{|c|c|c|c|c|c|c|c|}
\hline & $0 \% A V s$ & $10 \% \mathrm{AVs}$ & $30 \% \mathrm{AVs}$ & $50 \% \mathrm{AVs}$ & $70 \% \mathrm{AVs}$ & 90\%AVs & $100 \% A V s$ \\
\hline No control & Baseline & Improvement & & & & & \\
\hline$\Pi(s)$ & 322 & $1 \%$ & $8 \%$ & $13 \%$ & $23 \%$ & $25 \%$ & $26 \%$ \\
\hline$\Pi \mathrm{TC}<5 \mathrm{~s}$ & 26 & $-50 \%$ & $-58 \%$ & $-131 \%$ & $-138 \%$ & $-177 \%$ & $-192 \%$ \\
\hline $\mathrm{CO}(\mathrm{mg})$ & $1.5 \times 10^{8}$ & $-8 \%$ & $-22 \%$ & $-18 \%$ & $-29 \%$ & $-30 \%$ & $-20 \%$ \\
\hline $\mathrm{CO} 2(\mathrm{mg})$ & $6 \times 10^{9}$ & $-5 \%$ & $-20 \%$ & $-27 \%$ & $-41 \%$ & $-48 \%$ & $-27 \%$ \\
\hline $\mathrm{HC}(\mathrm{mg})$ & $8.4 \times 10^{5}$ & $-8 \%$ & $-21 \%$ & $-20 \%$ & $-31 \%$ & $-33 \%$ & $-21 \%$ \\
\hline PMx (mg) & $1.3 \times 10^{5}$ & $-6 \%$ & $-22 \%$ & $-26 \%$ & $-42 \%$ & $-47 \%$ & $-24 \%$ \\
\hline NOx (mg) & $2.5 \times 10^{6}$ & $-6 \%$ & $-21 \%$ & $-26 \%$ & $-41 \%$ & $-46 \%$ & $-22 \%$ \\
\hline Fuel (ml) & $2.6 \times 10^{6}$ & $-5 \%$ & $-20 \%$ & $-27 \%$ & $-41 \%$ & $-48 \%$ & $-27 \%$ \\
\hline VSL & Improvement & & & & & & \\
\hline$\Pi(\mathrm{s})$ & $-5 \%$ & $0 \%$ & $6 \%$ & $7 \%$ & $12 \%$ & $20 \%$ & $22 \%$ \\
\hline$\pi C<5 s$ & $-12 \%$ & $-58 \%$ & $-68 \%$ & $-126 \%$ & $-145 \%$ & $-178 \%$ & $-190 \%$ \\
\hline $\mathrm{CO}(\mathrm{mg})$ & $4 \%$ & $-10 \%$ & $-12 \%$ & $-13 \%$ & $-19 \%$ & $-15 \%$ & $-18 \%$ \\
\hline $\mathrm{CO} 2(\mathrm{mg})$ & $1 \%$ & $-5 \%$ & $-14 \%$ & $-23 \%$ & $-39 \%$ & $-40 \%$ & $-34 \%$ \\
\hline $\mathrm{HC}(\mathrm{mg})$ & $4 \%$ & $-9 \%$ & $-12 \%$ & $-15 \%$ & $-22 \%$ & $-19 \%$ & $-20 \%$ \\
\hline PMx (mg) & $5 \%$ & $-2 \%$ & $-8 \%$ & $-15 \%$ & $-30 \%$ & $-28 \%$ & $-22 \%$ \\
\hline $\mathrm{NOx}(\mathrm{mg})$ & $1 \%$ & $-6 \%$ & $-14 \%$ & $-23 \%$ & $-38 \%$ & $-37 \%$ & $-30 \%$ \\
\hline Fuel (ml) & $1 \%$ & $-5 \%$ & $-14 \%$ & $-23 \%$ & $-39 \%$ & $-40 \%$ & $-34 \%$ \\
\hline
\end{tabular}


willing to perform regulatory and cooperative changes rather than overtaking. AVs also encourage shorter timegaps between vehicles. Under free-flow conditions, the impact of AVs is not significant because there is no congestion formed. Meanwhile, VSL provides an efficient way to enhance the mobility, safety and environmental gains of the mixed flow when less than $70 \% \mathrm{AVs}$ are present. Nevertheless, for the ideal scenario that $90 \%$ or more AVs are on motorways, VSL is not triggered because solely AVs can achieve a non-congested traffic flow.

In order to explore the maximum possible benefits that AVs can provide, we increase the heavily congested traffic volume by 3 times. The results reveal that $100 \%$ AVs can lead to $83 \%, 88 \%, 26 \%$ improvements in total throughputs, maximum volume and travel time compared against that of $0 \% \mathrm{AVs}$ respectively. However, safety and environmental degradation because of deploying AVs are clearly observed. The reason for this degradation might be that increased throughputs result in higher density and closer time gaps between vehicles. Although, implementing VSL exerts negative impacts on the safety and mobility performance of the selected motorway, VSL relieves the increase in emissions and fuel consumption that is caused by accommodating additional vehicles.

The findings of this research work indicate that with existing traffic demands, the benefits of AVs are not remarkable at the initial stage of deployment. It is suggested to apply appropriate motorway control strategies on the mixed traffic flow with low AV shares. When $70 \%$ or more AVs are present, VSL has no impact on motorway mobility and safety. Thus, VSL can be implemented for other purposes (i.e., adverse weather, work zone) rather than improving mobility and safety if relatively high AV shares are achieved. In the future, the increased capacity because of deploying AVs may, in turn, encourage the increase in traffic demand. These additional vehicles may cause new safety and environmental issues. Existing VSL systems are not effective to deal with this extremely high traffic demands. Therefore, suitable motorway control tools should be developed taking into account the features of AVs as well as possible rise in demands. Encouraging shared AVs to replace privately owned vehicles can also handle the demand issue that we may encounter in the future. It should be noted that this study was conducted based on simulation. Nevertheless, the simulation model can have its own limitations to represent real-world traffic conditions. For example, AVs and HDVs were realized in simulation via setting different model parameters (e.g., minimum timegaps, speed factor, driver imperfection), whereas there are other differences between these two vehicle types that cannot be reflected in SUMO simulator. Thus, the findings of this study should be verified in the field when AVs are available to the mass market.

\section{Acknowledgements}

Not applicable.

\section{Authors' contributions}

$\mathrm{DL}$ did all the simulation experiments. The text is a joint work of all authors. All authors read and approved the final manuscript.

\section{Funding}

This study was sponsored by the Alexander von Humboldt Foundation, Natural Science Basic Research Plan in Shaanxi Province of China (Project No. 2017JQ5033), China Postdoctoral Science Foundation funded project (Project No. 2017M620434) Shaanxi Province Postdoctoral Science Foundation funded project (Project No. 2017BSHEDZZ38), and the Fundamental Research Funds for the Central Universities (Project No. 300102218309, No. 300102218401, No. 300102218404).

\section{Availability of data and materials}

The data that support the findings of this study are available from New Zealand Transport Agency (NZTA) but restrictions apply to the availability of these data, and so are not publicly available. Data are however available from the authors upon reasonable request and with permission of NZTA.

\section{Competing interests}

The authors declare that they have no competing interests.

Received: 3 October 2018 Accepted: 3 July 2019

Published online: 09 July 2019

\section{References}

1. Allaby, P., Hellinga, B., \& Bullock, M. (2007). Variable speed limits: Safety and operational impacts of a candidate control strategy for freeway applications. IEEE Transactions on Intelligent Transportation Systems, 8(4), 671-680.

2. Alvarez Lopez, P., Behrisch, M., Bieker-Walz, L., Erdmann, J., Flötteröd, Y., Hilbrich, R., Lücken, L., Rummel, J., Wagner, P., \& Wießner, E. (2018). Microscopic traffic simulation using SUMO, the 21st IEEE international conference on intelligent transportation systems, Maui, USA.

3. Bose, A., \& loannou, P. (2003). Analysis of traffic flow with mixed manual and semiautomated vehicles. IEEE Transaction on Intelligent Transportation Systems, 4, 173-188.

4. Brown, A., Gonder, J., \& Repac, B. (2014). In G. Meyer \& S. Beiker (Eds.), An analysis of possible energy impacts of autonomous vehicles. Road vehicle automation. Switzerland: Springer.

5. Cassidy, M. J., \& Rudjanakanoknad, J. (2015). Increasing the capacity of an isolated merge by metering its on-ramp. Transportation Research Part B: Methodological, 39(10), 896-913.

6. Chiou, Y. C., Huang, Y. F., \& Lin, P. C. (2012). Optimal variable speed-limit control under abnormal traffic conditions. Journal of the Chinese Institute of Engineers, 35(3), 299-308

7. Carlson, R., Papamichail, I., \& Papageorgiou, M. (2011). Local feedback-based mainstream traffic flow control on motorways using variable speed limits. IEEE Transactions on Intelligent Transportation Systems, 12(4), 1261-1276.

8. Davis, L. C. (2007). Effect of adaptive cruise control systems on mixed traffic flow near an on-ramp. Physica A: Statistical Mechanics and Its Applications, 379(1), 274-290.

9. Dowling, R., Skabardonis, A., Halkias, J., McHale, G., \& Zammit, G. (2004). Guidelines for calibration of micro-simulation models: Framework and applications. Transportation Research Record, 1876, 1-9.

10. Elefteriadou, L., Washburn, S., Yin, Y., Modi, Y., Letter, C. (2012) Variable speed limit (VSL) - Best management practice. Final report to the Florida department of transportation research center.

11. Erdmann, J. (2015). Chap. SUMO's lane-changing model. In M. Behrisch \& M. Weber (Eds.), Modeling mobility with open data. Modeling mobility with open data (pp. 105-123). Cham: Springer International Publishing.

12. Fagnant, D. J., \& Kockelman, K. (2015). Preparing a nation for autonomous vehicles: Opportunities, barriers and policy recommendations. Transportation Research Part A: Policy and Practice, 77, 167-181.

13. Hausberger, S., Krajzewicz, D. (2014) COLOMBO deliverable 4.2: Extended simulation tool PHEM coupled to SUMO with user guide. Projekt report. Deliverable 4.2, 61 pages, online at https://elib.dlr.de/98047/1/COLOMBO_ D4.2 ExtendedPHEMSUMO_v1.7.pdf

14. Hayes, B. (2011). Leave the driving to it. American Scientist, 99(5), 362-366. 
15. Habtemichael, F., \& Picado-Santos, L. (2013). Safety and operational benefits of variable speed limit under different traffic conditions and driver compliance levels. In 92nd annual meeting of transportation research board, Washington D.C.

16. Han, Y., Hegyi, A., Yuan, Y., Hoogendoorn, S., Papageorgiou, M., \& Roncoli, C. (2017). Resolving freeway jam waves by discrete first-order model-based predictive control of variable speed limits. Transportation Research Part C: Emerging Technologies, 77, 405-420.

17. Hayward, J. C. (1972). Near miss determination through use of a scale of danger. Report no. TTSC 7115. Pennsylvania: The Pennsylvania State University.

18. Hegyi, A., Hoogendoorn, S., Schreuder, M., Stoelhorst, H., \& Viti, F. (2008). SPECIALIST: A dynamic speed limit control algorithm based on shock wave theory. In IEEE conference on intelligent transportation systems, proceedings, ITSC'08 (pp. 827-832). Beijing: IEEE.

19. Hegyi, A., Schutter, B. D., \& Hellendoorn, H. (2005). Model predictive control for optimal coordination of ramp metering and variable speed limits. Transportation Research Part C, 13, 185-209.

20. Jerath, K., \& Brennan, S. (2010). Adaptive cruise control: Towards higher traffic flows, at the cost of increased susceptibility to congestion. In Proceedings of AVEC (Vol. 10). Loughborough: ACM.

21. Jin, H. Y., \& Jin, W. L. (2015). Control of a lane-drop bottleneck through variable speed limits. Transportation Research Part C: Emerging Technologies, $58,568-584$

22. Khondaker, B., \& Kattan, T. (2015). Variable speed limit: A microscopic analysis in a connected vehicle environment. Transportation Research Part C: Emerging Technologies, 58, 146-159.

23. Kesting, A., Treiber, M., \& Helbing, D. (2010). Enhanced intelligent driver model to access the impact of driving strategies on traffic capacity. Philosophical Transactions of the Royal Society of London A: Mathematical, Physical and Engineering Sciences, 368(1928), 4585-4605.

24. Kesting, A., \& Treiber, M. (2008). How reaction time, update time, and adaptation time influence the stability of traffic flow. Computer-Aided Civil and Infrastructure Engineering, 23(2), 125-137.

25. Krajzewicz, D., Erdmann, J., Behrisch, M., \& Bieker, L. (2012). Recent development and applications of SUMO - simulation of urban mobility. International Journal on Advances in Systems and Measurements, 5(3\&4), 128-138.

26. Krauß, S. (1998). Microscopic modeling of traffic flow: Investigation of collision free vehicle dynamics. Koln: PhD thesis, Mathematisches Institut, Universitat zu Koln, Germany: Hauptabteilung Mobilitat und Systemtechnik des DLR.

27. Li, D., Ranjitkar, P., Zhao, Y., Bai, Q., \& Chen, X. (2016). Improving motorway performance using variable speed limits. Proceedings of the Institution of Civil Engineers - Transport, 170(6), 338-345.

28. Li, D., \& Ranjitkar, P. (2015). A fuzzy logic-based variable speed limit controller. Journal of Advanced Transportation, 49(8), 913-927.

29. Martens, M., \& Brouwer, R. (2011). Linking behavioural indicators to safety: What is safe and what is not? In Proceedings of the 3rd international conference on road safety and simulation (pp. 1-14). Indianapolis: TRID.

30. Morrow, W. R., Greenblatt, J. B., Sturges, A., Saxena, S., Gopal, A., Millstein, D., Shah, N., \& Gilmore, E. A. (2014). In G. Meyer \& S. Beiker (Eds.), Key factors influencing autonomous vehicles' energy and environmental outcome. Road vehicle automation. Switzerland: Springer.

31. Müller, E. R., Carlson, R. C., \& Kraus, W. J. (2016). Cooperative mainstream traffic flow control on freeways. IFAC-Papers On Line, 49(32), 89-94.

32. National Highway Traffic Safety Administration. (2013). Preliminary statement of policy concerning automated vehicles. Washington, D.C: U.S. Department of Transportation.

33. Papageorgiou, M., Kosmatopoulos, E., \& Papamichail, I. (2008). Effects of variable speed limits on motorway traffic flow. Transportation Research Record, 2047, 37-48.

34. Papageorgiou, M., \& Papamichail, I. (2007). Handbook of ramp metering. Deliverable 7.5 (p. 2007). Brussels: Report for the European IST Office.

35. Perraki, G., Roncoli, C., Papamichail, I., \& Papageorgiou, M. (2018). Evaluation of a model predictive control framework for motorway traffic involving conventional and automated vehicles. Transportation Research Part C: Emerging Technologies, 92, 456-471.

36. Shladover, S., Su, D., \& Lu, X. (2012). Impacts of cooperative adaptive cruise control on freeway traffic flow. In Proceedings of the $91^{\text {st }}$ annual meeting of the transportation research board, Washington, D.C.

37. Tientrakool, P. Ho, Y., Maxemchuk, N. F. (2011) Highway capacity benefits from using vehicle-to-vehicle communication and sensors for collision avoidance. Vehicular Technology Conference.
38. Van den Hoogen, E., \& Smulders, S. (1994). Control by variable speed signs: Results of the Dutch experiment. In 7th international conference on road traffic monitoring and control (CP391) (pp. 145-149). London: IET.

\section{Publisher's Note}

Springer Nature remains neutral with regard to jurisdictional claims in published maps and institutional affiliations.

\section{Submit your manuscript to a SpringerOpen ${ }^{\circ}$ journal and benefit from:}

- Convenient online submission

- Rigorous peer review

- Open access: articles freely available online

High visibility within the field

- Retaining the copyright to your article

Submit your next manuscript at $\boldsymbol{\nabla}$ springeropen.com 